\title{
Synergistic effects of levosimendan and convalescence plasma as bailout strategy in acute cardiogenic shock in COVID-19: A case report
}

\author{
Henrik Fox ${ }^{1,2}$, Jan F. Gummert ${ }^{1,2}{ }^{(0)}$, Philipp Sommer ${ }^{2,4}$, \\ Cornelius Knabbe ${ }^{3}$, Christian Sohns ${ }^{4}$ (D) \\ ${ }^{1}$ Clinic for Thoracic and Cardiovascular Surgery, Herz- und Diabeteszentrum NRW, \\ Ruhr-Universität Bochum, Bad Oeynhausen, Germany \\ ${ }^{2}$ Heart Failure Department, Herz- und Diabeteszentrum NRW, \\ Ruhr-Universität Bochum, Bad Oeynhausen, Germany \\ ${ }^{3}$ Institute for Laboratory and Transfusion Medicine, Herz- und Diabeteszentrum NRW, \\ Ruhr-Universität Bochum, Bad Oeynhausen, Germany \\ ${ }^{4}$ Clinic for Electrophysiology, Herz- und Diabeteszentrum NRW, \\ Ruhr-Universität Bochum, Bad Oeynhausen, Germany
}

\section{Introduction}

Novel severe acute respiratory syndrome coronavirus 2 (SARS-CoV-2) has been described to induce a variety of clinical conditions [1]. While some patients have flu-like symptoms only, others develop serious coronavirus disease 2019 (COVID-19), which has been associated with high inflammatory burden including vascular inflammation, myocarditis and cardiac arrhythmias that can result in cardiogenic shock (CS) [1]. CS in general is still a challenging disease [2], but in the context of COVID-19 associated CS, mortality is especially high and treatment recommendations or promising strategies are lacking.

Compassionate use of convalescence plasma is essayed in multiple centers today, but convalescence plasma alone does not comprise immediate CS stabilization. It has capabilities to prevent severe COVID-19 outbreak [3], but in COVID-19 associated CS many physicians are left blanked-faced because of widely lacking evidence.

Utilization of catecholamines in CS is accompanied by side effects and the use of mechanical circulatory support in CS is associated with risk of infection, bleeding, vessel or nerve injury [4].
Therefore, substitute and new therapeutic options are warranted, especially to manage hypoperfusion and concomitant organ failure [5]. Calcium sensitizer levosimendan was developed to enhance inotropy [2], but levosimendan is under debate because available trials do not reflect the initial drug's promises [5], while no trial has tested the impact of levosimendan in COVID-19 associated CS to date.

In this context, reported herein, is a first case on a breakthrough of COVID-19 induced CS using synergistic effects of levosimendan in addition to convalescence plasma therapy in an 84-year-old.

\section{Case presentation}

An 84-year-old female with cardiovascular disease, including transcatheter aortic valve replacement in 2019, permanent atrial fibrillation, heart failure and chronic renal failure was admitted complaining of shortness of breath, blood pressure 85/60 $\mathrm{mmHg}$, heart rate 113/min. Rapidly, she developed high fever, non-productive cough and coronavirus polymerase chain reaction was positive for novel coronavirus SARS-CoV-2. The patient received 3 preparations of convalescence plasma $(190-230 \mathrm{~mL})$

Address for correspondence: Christian Sohns, MD, FEHRA, Clinic for Electrophysiology, Herz- und Diabeteszentrum NRW, Ruhr-Universität Bochum, Bad Oeynhausen, Germany, e-mail: csohns@hdz-nrw.de

Received: 16.05.2021 Accepted: 4.08.2021 Early publication date: 30.09.2021

This article is available in open access under Creative Common Attribution-Non-Commercial-No Derivatives 4.0 International (CC BY-NC-ND 4.0) license, allowing to download articles and share them with others as long as they credit the authors and the publisher, but without permission to change them in any way or use them commercially. 
Table 1. Laboratory parameters in COVID-19 cardiogenic shock.

\begin{tabular}{|c|c|c|c|c|c|}
\hline \multirow[t]{2}{*}{ Laboratory parameters } & \multirow[b]{2}{*}{ Day 1} & \multicolumn{2}{|c|}{$\begin{array}{l}\text { Addition of levosimendan } \\
\text { in this COVID-19 patient }\end{array}$} & \multirow[b]{2}{*}{ Night 2} & \multirow[b]{2}{*}{ Day 3} \\
\hline & & Night 1 & Day 2 & & \\
\hline Troponin & 147 & 192 & 159 & 76 & 89 \\
\hline BUN $[\mathrm{mg} / \mathrm{dL}]$ & 94 & 128 & 123 & 75 & 72 \\
\hline Creatinine $[\mathrm{mg} / \mathrm{dL}]$ & 1.4 & 1.9 & 1.7 & 1.7 & 1.1 \\
\hline MDRD [mL/min] & 36 & 25 & 29 & 47 & 53 \\
\hline $\mathrm{GOT}[\mathrm{U} / \mathrm{L}]$ & 21 & 1540 & 560 & 607 & 537 \\
\hline GPT [U/L] & 8 & 649 & 275 & 438 & 400 \\
\hline GGT [U/L] & 105 & 239 & 93 & 160 & 150 \\
\hline LDH [U/L] & 246 & 1240 & 599 & 534 & 497 \\
\hline Bilirubin [mg/dL] & 2.23 & 1.54 & 3.32 & 1.03 & 0.93 \\
\hline Interleukin 6 [ng/L] & 79 & 205 & 98 & 40 & 35 \\
\hline NT-proBNP [pg/mL] & 12800 & 10800 & 10500 & 7290 & 2180 \\
\hline $\mathrm{CRP}[\mathrm{mg} / \mathrm{dL}]$ & 8.4 & 11 & 10 & 8.9 & 6.0 \\
\hline Procalcitonin [ng/mL] & $<0.2$ & $<0.2$ & $<0.2$ & $<0.2$ & $<0.2$ \\
\hline \multicolumn{6}{|l|}{ Blood gas analyses: } \\
\hline $\mathrm{pH}$ & 7.457 & 7.421 & 7.421 & 7.479 & 7.490 \\
\hline $\mathrm{pCO}_{2}[\mathrm{mmHg}]$ & 34.0 & 25.6 & 26.6 & 28.4 & 30.1 \\
\hline $\mathrm{HCO}_{3}[\mathrm{mmol} / \mathrm{L}]$ & 24.4 & 17.2 & 19.5 & 23.0 & 24.7 \\
\hline Base excess & -0.9 & -7.2 & -2.5 & -1.7 & -0.4 \\
\hline Lactate $[\mathrm{mmol} / \mathrm{L}]$ & 3.9 & 6.7 & 8.6 & 4.1 & 1.5 \\
\hline $\mathrm{CVPO}_{2}[\mathrm{mmHg}]$ & 34.6 & 26.1 & 43.2 & 46.8 & 53.8 \\
\hline
\end{tabular}

BUN - blood urea nitrogen; CRP - C-reactive protein; $\mathrm{CVPO}_{2}$ - central venous oxygen saturation; GGT — gamma-glutamyl transpeptidase; GOT - glutamic-oxaloacetic transaminase; GPT — glutamic-pyruvic transaminase; $\mathrm{HCO}_{3}$ — bicarbonate; LDH — lactate dehydrogenase; MDRD - modification of diet in renal disease; NT-pro BNP - N-terminal pro-B-type natriuretic peptide; $\mathrm{pCO}_{2}-$ partial pressure of carbon dioxide; $\mathrm{pH}-\mathrm{pH}$, decimal logarithm of the reciprocal of the hydrogen ion activity

on day 1, 3 and 5 after preexistent IgA and $\operatorname{IgG}$ antibodies had been excluded (anti-SARS-CoV-2-ELISA, EUROIMMUN AG, Lübeck, Germany).

Only hours after hospital admittance her hemodynamics rapidly deteriorated and she developed CS with severely impaired left ventricular function (LVEF 30\%). Coronary angiography excluded coronary artery disease, developing COVID-19 associated CS.

Her clinical condition progressively deteriorated despite optimal guideline-derived CS medical treatment [2], with dyspnea at rest, tachypnea, orthopnea and cyanosis, requiring rising doses of intravenous inotrope drugs (dobutamine $8 \mu \mathrm{g} / \mathrm{kg}$ / $/ \mathrm{min}$, norepinephrine $0.5 \mu \mathrm{g} / \mathrm{kg} / \mathrm{min}$ ). She developed systemic hypoperfusion syndrome resulting in multiple organ failure, high serum lactate levels and central venous oxygen saturation of $30 \%$ (Table 1).

At this time, available CS treatment options were exhausted and for further aggravation of COVID-19 induced CS, levosimendan was applied as a bailout compassionate therapy. After that, improvement in hemodynamics and clinical parameter eventuated (Table 1). Lactic acidosis came to regression and multiorgan failure slowly reversed. Given the life-threatening COVID-19 associated $\mathrm{CS}$ condition in this patient, add-on treatment with intravenous levosimendan $2.5 \mathrm{mg}(12 \mu \mathrm{g} / \mathrm{kg}$ as bolus over $10 \mathrm{~min}$ and $0.1 \mu \mathrm{g} / \mathrm{kg} / \mathrm{min}$ as infusion) adjunctive to convalescence plasma therapy resulted in improvement and finally breakthrough of COVID-19 associated CS, including clinical improvements, such as relief from dyspnea and orthopnea.

\section{Discussion}

According to available research, herein, is the first clinical in-vivo observation reported using the combined application of convalescence plasma and novel calcium sensitizer levosimendan to overcome acute CS in a COVID-19 patient. After 5 days of treatment the patient was stable enough to be discharged from intensive care unit and weeks after hospital admission she returned to her ordinary daily life. 
We describe a novel treatment strategy for a complex clinically new disease pattern, with no verified therapy scheme known so far. During this life-threatening condition additional use of levosimendan resulted in potential synergistic effects resulting in hemodynamic stabilization and our strategy overcame CS not requiring invasive mechanical circulatory support (MCS), as MCS invasiveness in an 84-year-old carries notable risks such as bleeding, infection or thromboembolic complications $[4,6]$.

Moreover, levosimendan is currently the subject of intense discussion too, as this novel drug promises positive inotropic effects, but large clinical trials failed to confirm these effects [7]. European Society of Cardiology (ESC) guidelines recommend levosimendan in acute heart failure only to reverse effects of beta-blockade, if beta-blockade is considered to contribute to CS (evidence class IIb, level C) [2]. Furthermore, the drug may contribute to severe arrhythmia, myocardial ischemia and hypotension (evidence class I, level C). ESC guidelines recommend levosimendan only in combination with other established inotropes, such as dobutamine or vasopressors in CS [2] and levosimendan has undesired effects such as vasodilation and arrhythmia. In addition to that current goal in COVID-19 treatment is to reduce intravascular fluids to avoid mechanical ventilation while CS treatment often requires intravascular fluids. This relation seems incompatible and hemodynamic monitoring is challenging [8], why levosimendan use can only be understood as a bailout strategy.

Large randomized controlled trials such as the CHEETAH study investigated hemodynamic effects of levosimendan in 506 high-risk patients undergoing cardiac surgery, finding no benefit in terms of mortality or other clinical endpoints [5]. Similarly, the LEVO-CTS trial [9] or the LeoPARDS trial [10] found no benefit in comparison to placebo.

Besides available evidence, there is no randomized controlled clinical trial, nor any clinical evidence on how to manage therapy-refractory COVID-19 CS, because our first in-vivo observation of synergistic effects for the combination of levosimendan and plasma therapy brings the hypothesis that levosimendan may be an option in critical COVID-19 induced CS [5]. Synergistic effects may derive from the timely critical combination of the immune system response through convalescence plasma therapy, but which requires hemodynamic stability and sufficient circulation that may have been provided through levosimendan application. This engaged synergy appears to have facilitation of breakthrough of COVID-19 CS in our presented case.
However, additional research is needed to better define indications for, and benefits of, levosimendan therapy. Nevertheless, studies of COVID-19 CS patients are difficult to conduct, but scientific data is important to provide assistance on how to best treat COVID-19 CS patients and whether levosimendan application in combination with other therapeutics might lead to similar benefits as observed in our reported case.

\section{Ethics statement}

An exemption of ethical approval has been assigned for reporting this case and this report is in accordance with the Declaration of Helsinki.

\section{Acknowledgments}

We acknowledge support by the Open Access Publication Funds of the Ruhr-Universität Bochum.

\section{Conflict of interest: None declared}

\section{References}

1. Madjid M, Safavi-Naeini P, Solomon SD, et al. Potential effects of coronaviruses on the cardiovascular system: a review. JAMA Cardiol. 2020; 5(7): 831-840, doi: 10.1001/jamacardio.2020.1286, indexed in Pubmed: 32219363.

2. Ponikowski P, Voors AA, Anker SD, et al. 2016 ESC Guidelines for the diagnosis and treatment of acute and chronic heart failure: The Task Force for the diagnosis and treatment of acute and chronic heart failure of the European Society of Cardiology (ESC). Developed with the special contribution of the Heart Failure Association (HFA) of the ESC. Eur Heart J. 2016; 37(27): 2129-2200, doi: 10.1093/eurheartj/ehw128, indexed in Pubmed: 27206819.

3. Roback JD, Guarner J. Convalescent plasma to treat COVID-19: possibilities and challenges. JAMA. 2020; 323(16): 1561-1562, doi: 10.1001/jama.2020.4940, indexed in Pubmed: 32219429.

4. Gyoten T, Rojas SV, Irimie A, et al. Patients with ventricular assist device and cerebral entrapment-Supporting skullcap reimplantation. Artif Organs. 2021; 45(5): 473-478, doi: 10.1111/ aor.13856, indexed in Pubmed: 33141976.

5. Maack C, Eschenhagen T, Hamdani N, et al. Treatments targeting inotropy. Eur Heart J. 2019; 40(44): 3626-3644, doi: 10.1093/eurheartj/ehy600, indexed in Pubmed: 30295807.

6. Gyoten T, Morshuis M, Rojas SV, et al. Identification of characteristics, risk factors, and predictors of recurrent LVAD thrombosis: conditions in HeartWare devices. J Artif Organs. 2021; 24(2): 173-181, doi: 10.1007/s10047-020-01228-2, indexed in Pubmed: 33337536.

7. Flottmann C, Dumitrescu D, Sohns C, et al. Addition of levosimendan to overcome acute cardiogenic shock - Paving the way for later heart transplantation. A first case report. Clin Case Rep. 2021; 9(2): 856-860, doi: 10.1002/ccr3.3672, indexed in Pubmed: 33598259.

8. Szymczyk T, Sauzet O, Paluszkiewicz LJ, et al. Non-invasive assessment of central venous pressure in heart failure: a systematic prospective comparison of echocardiography and Swan-Ganz catheter. Int J Cardiovasc Imaging. 2020; 36(10): 1821-1829, doi: 10.1007/s10554-020-01889-3, indexed in Pubmed: 32445006.

9. Mehta R, Leimberger J, Diepen Sv, et al. Levosimendan in patients with left ventricular dysfunction undergoing cardiac surgery. N Engl J Med. 2017; 376(21): 2032-2042, doi: 10.1056/ nejmoa1616218.

10. Gordon A, Perkins G, Singer M, et al. Levosimendan for the Prevention of Acute Organ Dysfunction in Sepsis. N Engl J Med. 2016; 375(17): 1638-1648, doi: 10.1056/nejmoa1609409. 